\title{
Controls on ERS altimeter measurements over ice sheets: Footprint-scale topography, backscatter fluctuations, and the dependence of microwave penetration depth on satellite orientation
}

\author{
R. J. Arthern, ${ }^{1}$ D. J. Wingham, and A. L. Ridout \\ Department of Space and Climate Physics, University College London, London, England, UK
}

\begin{abstract}
We consider the reliability of radar altimeter measurements of ice sheet elevation and snowpack properties in the presence of surface undulations. We demonstrate that over ice sheets the common practice of averaging echoes by aligning the first return from the surface at the origin can result in a redistribution of power to later times in the average echo, mimicking the effects of microwave penetration into the snowpack. Algorithms that assume the topography affects the radar echo shape in the same way that waves affect altimeter echoes over the ocean will therefore lead to biased estimates of elevation. This assumption will also cause errors in the retrieval of echoshape parameters intended to quantify the penetration of the microwave pulse into the snowpack. Using numerical simulations, we estimate the errors in retrievals of extinction coefficient, surface backscatter, and volume backscatter for various undulating topographies. In the flatter portions of the Antarctic plateau, useful estimates of these parameters may be recovered by averaging altimeter echoes recorded by the European Remote Sensing satellite (ERS-1). By numerical deconvolution of the average echoes we resolve the depths in the snowpack at which temporal changes and satellite traveldirection effects occur, both of which have the potential to corrupt measurements of ice sheet elevation change. The temporal changes are isolated in the surface-backscatter cross section, while directional effects are confined to the extinction coefficient and are stable from year to year. This allows the removal of the directional effect from measurement of ice-sheet elevation change.
\end{abstract}

\section{Introduction}

Satellite radar altimetry is being used to measure the mass balance of the large ice sheets covering Greenland and Antarctica [Zwally et al., 1989; Davis et al., 1998; Wingham et al., 1998]. To improve upon ground-based estimates of mass balance, measurements of elevation change, accurate to a few centimeters per year, are needed. A common technique is to compare elevations measured at crossover points, where an earlier satellite ground track is crossed by a later one [Zwally et al., 1989]. Changes in the shape of the radar echo (i.e., the shape of return power plotted as a function of time delay since pulse transmission) can cause errors in these measurements and must be investigated before one can attribute physical significance to measurements of ice-sheet elevation change recorded by radar altimeters. In this paper we describe such an investigation.

Brown [1977] modeled the expected echo shape for altimeter returns from the ocean surface, but there are two important differences between altimeter echoes from ice sheets and from oceans. Firstly, the ice sheets are much more undulating than the oceans, and this topography can affect the shape of the

\footnotetext{
${ }^{1}$ Now at British Antarctic Survey, Natural Environment Research Council, High Cross, Cambridge, England, UK.

Copyright 2001 by the American Geophysical Union.

Paper number 2001JD000498.

0148-0227/01/2001JD000498S09.00
}

echo [Brenner et al., 1983; Martin et al., 1983; Wingham, 1995; Legrésy and Rémy, 1997]. Secondly, the Ku-band and C-band microwave pulses transmitted by most spacebourne altimeters can penetrate several meters into the snowpack before being reflected back to the satellite [Ridley and Partington, 1988; Davis and Moore, 1993; Legrésy and Rémy, 1998].

Both topography and penetration affect the elevation distribution of scatterers. Wingham [1995] showed that a simple convolution operator relates the distribution of scatterer elevations to the average altimeter echo recorded over a region, provided that the origin of time delay is shifted to coincide with the arrival of a hypothetical reflection from a spherical datum surface, rather than the moment of pulse transmission. In practice, however, this procedure has not been followed: in studying the average echo shape, Ridley and Partington [1988], Partington et al. [1989], Davis and Moore [1993], and Yi et al. [1997] have chosen the origin of time delay to correspond to the first arrival from the actual surface, rather than a predetermined, spherical datum. We will call this procedure "firstarrival averaging" to distinguish it from the "datum-arrival averaging" considered by Wingham [1995]. The effect of footprint-scale topography upon average echoes obtained by firstarrival averaging has not been investigated. Ridley and Partington [1988], Partington et al. [1989], Davis and Moore [1993], and Yi et al. [1997] each assume that the topography will affect the average echo in the same way that waves affect the shape of echoes recorded over the ocean, i.e., a broadening of the echo by convolution with a height distribution which may be as- 
sumed close to Gaussian in shape. In sections 2 and 3 of this paper we investigate the validity of this assumption.

In section 2 we show that although the effect of footprintscale topography upon the average echo obtained by firstarrival averaging can be described by a convolution operator, the kernel of this operator bears no simple relation to the height distribution of ice sheet topography. Furthermore, for all but the flattest terrain, this kernel will be poorly approximated by a Gaussian. This has several implications. (1) It may be much more difficult to separate the effects of topography and penetration upon echo shape than has previously been recognized. (2) Parameters describing microwave penetration and scattering (including the surface backscatter, volume backscatter, and the extinction coefficient) may be in error when recovered from echoes recorded over undulating terrain. (3) Retracking algorithms that assume a Gaussian convolution model for the effect of topography upon echo shape will, in general, give biased results, with the bias becoming increasingly severe as the surface topography becomes more rugged; and (4) measurements of ice sheet elevation change using such algorithms are likely to be incorrect if the microwavescattering properties of the snowpack change over time.

The severity of the problems listed above will depend upon the amplitude and wavelength of undulations. In section 3 we perform numerical calculations to quantify the errors that may result from undulations with amplitudes and horizontal scales typical of ice sheets. In section 4 we document backscatter and echo shape variations in Antarctica, limiting our investigation to regions of thick ice where the ice surface is relatively flat, and our numerical simulations suggest the effects of penetration may be distinguished from those of topography. We use echoes recorded by the ERS-1 altimeter (a nadir-pointing, linearly polarized, Ku-band radar), paying particular attention to temporal changes in echo shape and to discrepancies between ascending passes (northward satellite motion) and descending (southward) passes. We compare our retrievals with previous inversions of echo shape [Legrésy and Rémy, 1998, 1999]. Using numerical deconvolution methods, we are able to resolve temporal changes in backscatter in time delay and show that these are confined to the air-snow interface (in agreement with Legrésy and Rémy [1998]), while direction-dependent effects are confined to the extinction coefficient (in contrast to Legrésy and Rémy [1999] who attributed them to the volume backscatter cross section). This separation provides independent confirmation of our ability to separate topography and penetration effects in the average echo. In section 5 we discuss the implications of our findings for measurements of ice sheet mass balance using satellite radar altimetry.

\section{Theory}

In this section we derive an expression for a convolution operator, with kernel $D_{\text {long, }}$, which describes the effect of footprint-scale undulations upon the echo shape obtained by firstarrival averaging. Secondly, we demonstrate that this kernel is poorly approximated by a Gaussian for all but the flattest terrain. In doing so, we shall also introduce mathematical operators, which form the basis for our processing of ERS-1 radar altimeter echoes in section 4.

Wingham [1995] has developed a general expression for the altimeter echo over a surface having undulations typical of those encountered on ice sheets. This derivation, which is too lengthy to describe in full detail here, is essentially a linerari-

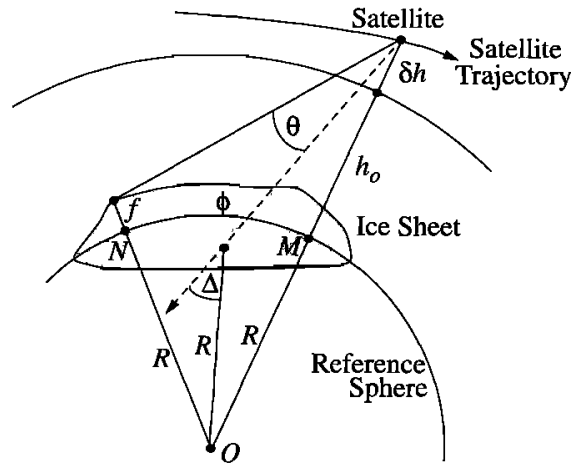

Figure 1. Coordinates used in the derivation of the altimeter echo. All elevations $f(N)$ and the satellite altitude $h_{0}+$ $\delta h(M)$ are referenced to a sphere of radius $R$ centered at $O$. $R$ and $O$ are chosen such that the reference sphere is approximately coincident with the ice sheet surface. Points $N$ and $M$ lying on this sphere define the position of the scattering point and the satellite, respectively. $N$ and $M$ subtend an angle $\phi$ at $O$, and the scattering point lies at angle $\theta$ from the antenna boresight (dashed line). The boresight intercepts the reference sphere at an angle $\Delta$ to its normal. Note that the boresight direction need not lie in the plane NOM.

zation of the radar equation [Skolnik, 1970], integrated over surface and volume-scattering elements within the snowpack. For the geometry shown in Figure 1, the resulting expression is

$$
\begin{aligned}
p(M, t) \approx & \frac{E_{t} \lambda^{2}}{(4 \pi)^{3} h_{0}^{4} L_{s}} \int_{-\infty}^{\infty} d \tau s(t-\tau) \\
& \cdot \iint_{N \in \text { Sphere }} d S_{N} \cdot g^{2}\left(\frac { 2 R } { h _ { 0 } } \left[\sin ^{2} \frac{\phi}{2}+\frac{h_{0}^{2}}{R^{2} \eta^{2}} \sin ^{2} \frac{\Delta}{2}\right.\right. \\
& \left.\left.-\frac{h_{0} \sin \Delta}{2 R \eta} \sin \phi \cos \left(\rho-\rho_{\mathrm{SM}}\right)\right]^{1 / 2}\right) \\
& \cdot q\left(\tau-\frac{4 R^{2} \eta}{c h_{0}} \sin ^{2} \frac{\phi}{2}+\frac{2 f(N)}{c}-\frac{2 h_{0}}{c}-\frac{2 \delta h(M)}{c}\right)
\end{aligned}
$$

where $E_{t} q(t)$ is the effective transmitted-power envelope as a function of time $t$, and $E_{t}$ is the total energy transmitted during each pulse; $c$ is the speed of light in vacuum; $\lambda$ is the wavelength of the transmitted radiation; $g(\sin \theta)$ is the gain of the antenna at angle $\theta$ to the boresight direction (dashed line in Figure 1); $L_{s}$ represents the power loss incurred in propagation through the atmosphere; $\phi$ is the angle subtended at $O$ by the points $N$ and $M$, where $\mathrm{O}$ is the center of a reference sphere, of radius $R$, which closely approximates the ice sheet surface; $N$ and $M$ are, respectively, the radial projections of the scattering point and the satellite location onto this sphere. The integration is performed over the sphere surface. The dimensionless constant $\eta$ accounts for the Earth's curvature and is given by

$$
\eta=\left(1+\frac{h_{0}}{R}\right) \text {. }
$$

The coordinates $[R, \rho, \phi]$ locate the scattering point $N$ in a spherical coordinate system, centered at $O$, and with axis of rotation along $O M ; \rho_{S M}$ is the azimuthal angle of the bore- 
sight direction in the same coordinate system, and $\Delta$ is the angle between the sphere normal and the boresight at the point where the boresight intersects the reference sphere (Figure 1). The satellite lies a distance $h_{0}+\delta h(M)$ from the point $M$, where the second term represents small variations about the approximate distance $h_{0}$. See Wingham [1995] for details of the assumptions used to derive equation (1).

The function $s$, which is not explicitly defined by Wingham [1995], but is useful to introduce here, is given by

$s(t)=\left\{\begin{array}{ll}\sigma_{\mathrm{surf}}^{\mathrm{o}} \delta(t)+\sigma_{\mathrm{vol}}^{\mathrm{o}} c_{\mathrm{ice}} k_{e} \exp \left[-c_{\mathrm{cee}} k_{e} t\right] & t \geq 0 \\ 0, & t<0\end{array}\right.$,

where $\sigma_{\text {surf }}^{\text {o }}$ is the backscatter coefficient of the surface; $\delta$ is the Dirac delta function, and the integrated volume backscatter $\sigma_{\mathrm{vol}}^{\mathrm{o}}$ is defined, as follows, to ensure that $\sigma_{\text {surf }}^{\mathrm{o}}+\sigma_{\mathrm{vol}}^{\mathrm{o}}=\sigma^{\mathrm{o}}$, the total backscatter.

$$
\sigma_{\mathrm{vol}}^{\mathrm{o}}=\frac{\sigma_{v} k_{t}^{2}}{2 k_{e}} .
$$

In equation (4), $\sigma_{v}$ is the radar cross section per unit volume of snow; $c_{\text {1ce }}$ is the speed of propagation within the snow; $k_{e}$ is the extinction coefficient, assumed constant within the range of depths sampled by the altimeter; and $k_{t}$ is the transmission coefficient of the air-snow interface. Physically, $s$ may be regarded as the distribution of backscatter with depth (expressed in terms of the propagation delay in the snow); the first term describes the reflection from the air-snow interface, the second term is the exponentially decaying contribution from the volume. There is no scattering from above the surface, so $s$ is a one sided function; that is, it is zero for negative propagation delays. Together, (1) to (4) comprise our model for the altimeter echo recorded by an altimeter above location $M$.

We now turn to the effect of topographic undulations upon the echo shape. To do this, it is useful to separate the topography $f$ into a short-scale component $f_{\text {short }}$, which varies on length scales of a few meters, much smaller than the altimeter footprint, and a remaining long-scale component $f_{\text {long, which }}$ includes the footprint-scale topography, so

$$
f=f_{\text {short }}+f_{\text {long }} \text {. }
$$

The effect of the short-scale topography upon the echo shape can be found by considering the quantity $u=-2 f_{\text {short }} / c$ to be a random variable with a probability density function $D_{\text {short }}$. Because $f_{\text {short }}$ varies on scales much shorter than the altimeter footprint, its effects are well averaged, even in a single echo. Substituting (5) into (1), and taking the expected value, gives

$$
\begin{aligned}
p(M, t) \approx & \frac{E_{t} \lambda^{2}}{(4 \pi)^{3} h_{0}^{4} L_{s}} \int_{-\infty}^{\infty} d \tau s(t-\tau) \\
& \cdot \int_{-\infty}^{\infty} d u D_{\text {short }}(u) \iint_{N \in \text { Sphere }} d S_{N} \\
& \cdot g^{2}\left(\frac { 2 R } { h _ { 0 } } \left[\sin ^{2} \frac{\phi}{2}+\frac{h_{0}^{2}}{R^{2} \eta^{2}} \sin ^{2} \frac{\Delta}{2}-\frac{h_{0} \sin \Delta}{2 R \eta}\right.\right. \\
& \left.\left.\cdot \sin \phi \cos \left(\rho-\rho_{\mathrm{SM}}\right)\right]^{1 / 2}\right) \cdot q\left(\tau-u-\frac{4 R^{2} \eta}{c h_{0}}\right. \\
& \left.\cdot \sin ^{2} \frac{\phi}{2}+\frac{2 f_{\text {long }}(N)}{c}-\frac{2 h_{0}}{c}-\frac{2 \delta h(M)}{c}\right) .
\end{aligned}
$$

In general, the longer-scale topography $f_{\text {long }}$ varies too slowly across the footprint for its effect upon the echo shape to be modeled as a well-averaged random process. We first consider the case when the topography varies only upon scales much larger than the altimeter footprint. Then, for all points $N$ where the antenna gain $g$ is nonnegligible,

$$
f_{\text {long }}(N) \approx f_{\text {long }}(M) \text {. }
$$

This allows the altimeter echo to be written as a convolution of four terms as follows:

$$
\begin{aligned}
p\left(M, t+t_{0}(M)\right) \approx & \int_{-\infty}^{\infty} d \tau s(t-\tau) \int_{-\infty}^{\infty} d u D_{\text {short }}(u) \\
& \cdot \int_{-\infty}^{\infty} d v q(v) I(\tau-v-u)
\end{aligned}
$$

where

$$
\begin{aligned}
I(t)= & \frac{E_{t} \lambda^{2}}{(4 \pi)^{3} h_{0}^{4} L_{s}} \iint_{N \in \text { Sphere }} d S_{N} \delta\left(t-\frac{4 R^{2} \eta}{c h_{0}} \sin ^{2} \frac{\phi}{2}\right) \\
& \cdot g^{2}\left(\frac { 2 R } { h _ { 0 } } \left[\sin ^{2} \frac{\phi}{2}+\frac{h_{0}^{2}}{R^{2} \eta^{2}} \sin ^{2} \frac{\Delta}{2}-\frac{h_{0} \sin \Delta}{2 R \eta}\right.\right. \\
& \left.\left.\cdot \sin \phi \cos \left(\rho-\rho_{\mathrm{SM}}\right)\right]^{1 / 2}\right)
\end{aligned}
$$

is the impulse response of the reference sphere per unit backscatter; it is essentially the impulse response of a flat surface calculated by Brown [1977] but with the effect of curvature included.

In (8) the origin of time delay has been redefined to correspond to the time of arrival $t_{0}(M)$ from the point on the surface $f_{\text {long }}$ closest to the satellite. This choice of origin defines first-arrival averaging. If the approximation (7) is satisfied, then

$$
t_{0}(M) \approx \frac{2 h_{0}}{c}+\frac{2 \delta h(M)}{c}-\frac{2 f_{\text {long }}(M)}{c}
$$

and (8) follows from equation (6).

We now consider the case when approximation (7) is not satisfied. Then the echo shape will be different from the simplified model defined by (8). In this more general case we have

$$
\begin{aligned}
p\left(M, t+t_{0}(M)\right) \approx & \int_{-\infty}^{\infty} d \tau s(t-\tau) \int_{-\infty}^{\infty} d u D_{\text {short }}(u) \\
& \cdot \int_{-\infty}^{\infty} d v q(v) I_{M}(M, \tau-v-u)
\end{aligned}
$$




$$
\begin{aligned}
I_{M}(M, t)= & \frac{E_{t} \lambda^{2}}{(4 \pi)^{3} h_{0}^{4} L_{s}} \iint_{N \in \text { Sphere }} d S_{N} \delta\left(t-\frac{4 R^{2} \eta}{c h_{0}} \sin ^{2} \frac{\phi}{2}\right. \\
& \left.+\frac{2 f_{\text {long }}(N)}{c}-\frac{2 h_{0}}{c}-\frac{2 \delta h(M)}{c}+t_{0}(M)\right) \\
& \cdot g^{2}\left(\frac { 2 R } { h _ { 0 } } \left[\sin ^{2} \frac{\phi}{2}+\frac{h_{0}^{2}}{R^{2} \eta^{2}} \sin ^{2} \frac{\Delta}{2}\right.\right. \\
& \left.\left.-\frac{h_{0} \sin \Delta}{2 R \eta} \sin \phi \cos \left(\rho-\rho_{\mathrm{SM}}\right)\right]^{1 / 2}\right)
\end{aligned}
$$

is the impulse response of the undulating surface $f_{\text {long. }}$. We can now introduce a convolution operator $D_{\text {long, }}$, which quantifies the departure of the echo shape predicted by (12) from that predicted by (8). In other words we require that

$$
\begin{aligned}
p\left(M, t+t_{0}(M)\right) \approx & \int_{-\infty}^{\infty} d \tau s(t-\tau) \int_{-\infty}^{\infty} d u D_{\text {short }}(u) \int_{-\infty}^{\infty} d v q(v) \\
& \cdot \int_{-\infty}^{\infty} d w D_{\text {long }}(M, w) I(\tau-v-u-w),
\end{aligned}
$$

which in turn requires that

$$
D_{\text {long }}(M, t)=\int_{-\infty}^{t} d \tau K_{r}(t-\tau) I_{M}(M, \tau),
$$

where the kernel $K_{r}$ satisfies $K_{r}(t)=0$, for all $t<0$, and

$$
\delta(t)=\int_{-\infty}^{t} d \tau K_{r}(t-\tau) I(\tau)
$$

otherwise. It is clear from (9), (12), (14), and (15), which together define $D_{\text {long, }}$, that this function bears no simple relation to the distribution of the long-wavelength topography in the way that $D_{\text {short }}$ does to the short-scale topography. In particular, $D_{\text {long }}$ depends functionally upon the altimeter beamwidth and satellite altitude as well as upon the geometry of the surface. Furthermore, because $t_{0}(M)$ is defined to be the time of arrival from the closest point on the surface $f_{\text {long, }}$, it follows that

$\frac{4 R^{2} \eta}{c h_{0}} \sin ^{2} \frac{\phi}{2}+\frac{2 f_{\text {long }}(N)}{c}-\frac{2 h_{0}}{c}-\frac{2 \delta h(M)}{c}+t_{0}(M)>0$

to the order of accuracy used to derive (1). Equation (16) can be used to show that $D_{\text {long }}(M, t)=0$ for all $t<0$. Clearly, $D_{\text {long }}$ cannot be Gaussian because it is a one-sided function. This implies that when first-arrival averaging is used, the longscale topography always causes the redistribution of power toward later times in the echo. Microwave penetration into the snow also skews the distribution of arrival time in favor of later arrivals, by convolution with the one-sided function $s$, so there is obviously scope for confusing the effects of penetration with the distortion of the echo introduced by the footprint-scale topography.

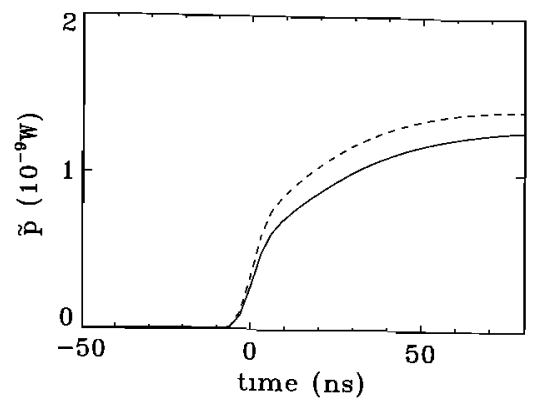

Figure 2. Average of 400 simulated echoes from $100 \times 100$ $\mathrm{km}$ computer-generated undulating surface (solid line). The surface undulations had a standard deviation $10 \mathrm{~m}$ and an autocorrelation length $5 \mathrm{~km}$, values representative of ice sheet undulations. The dashed line shows the average echo that would result with no undulations present.

\section{Influence of Footprint-Scale Topography on Spatially Averaged Echoes}

To see how serious the confusion between penetration and topography may become, it would be useful to calculate the kernel $D_{\text {long. }}$ When $f_{\text {long }}$ is constant, the approximation (7) holds everywhere with equality. Then $D_{\text {long }}(M, t)=\delta(t)$ and (8) and (13) are identical. In general, however, $D_{\text {long }}$ is a function of the particular topography within the altimeter footprint, and a different $D_{\text {long }}$ results from any particular topography. We performed numerical calculations to reveal the effects of topography upon the first-arrival-averaged echo $\bar{p}(t)$, defined as

$$
\bar{p}(t)=\iint_{\text {sphere }} d S_{M} W(M) p\left(M, t+t_{0}(M)\right),
$$

where the integration is performed over a localized region weighted by the function $W$ (see Wingham [1995] for the functional form of $W$ and the practical considerations determining its spatial scale). We consider the average echo, rather than individual echoes, because the shape of the spatial average defined by (17) is much less sensitive to the exact configuration of the topography within any given footprint, and also because, in practical situations, it is useful to reduce the speckle noise on individual echoes by averaging them.

Figure 2 shows a numerical calculation of $\bar{p}$ from a computer-generated $100 \mathrm{~km}$ by $100 \mathrm{~km}$ topography with an $\boldsymbol{e}$-fold decorrelation length of $5 \mathrm{~km}$ and a standard deviation of $10 \mathrm{~m}$ (similar to undulations observed in digital elevation models derived from interferometric radar [Joughin et al., 1996]). The average echo, formed from 400 simulated echoes on a 20 by 20 grid covering the region, shows the distortion introduced by the topography, compared to the case without undulations (dashed line). In both cases, sub-footprint-scale topography and penetration effects are incorporated by numerical convolution. The effect of the long-wavelength topography is included by summing the reflected power envelope from each of a rectangular grid of surface elements with appropriate time delays for the travel time between each surface element and the altimeter, as described by Féménias et al. [1993]. The grid spacing was $100 \mathrm{~m}$, and 1024 elements were used in each direction. For the case without topography, these elements were assigned to lie on the reference sphere. The numerical 


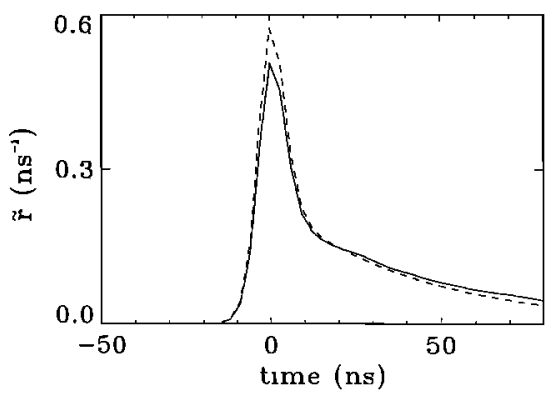

Figure 3. Deconvolution procedure removes slant-range effects, revealing the distribution of scattering with depth more clearly. The deconvolutions were derived from the echoes shown in Figure 2, with and without undulations (solid and dashed lines, respectively). The effect of undulations mimics a smaller reflection from the air-snow interface (delay times close to zero), more subsurface scattering (delay times greater than zero), and a shallower decay with depth. A delay of $80 \mathrm{~ns}$ corresponds to scattering from about $10 \mathrm{~m}$ in depth.

calculation for the case without undulations agreed with an analytical evaluation of (1) to within $1 \%$.

To reveal more clearly the contributions of surface and volume scattering, we employ a deconvolution method similar to that described by Lipa and Barrick [1981] for ocean echoes. Solution of the integral equation

$$
\bar{p}(t)=\int_{-\infty}^{t} d \tau I(t-\tau) \bar{r}(\tau)
$$

provides a function $\bar{r}(t)$, shown in Figure 3, which we refer to as the "deconvolution" of the echo. It is easier to see the contributions from reflection at the air-snow interface (sharp peak) and volume scattering (exponentially decaying tail) in the deconvolution than in the average echo. Note that because (18) is linear, solving for the deconvolution of the average echo is equivalent to deconvolving individual echoes and then averaging. Operating on the average echo is computationally more efficient, however, and is the procedure adopted throughout this paper.

As predicted in section 2, the undulations cause power to be redistributed to later times: the effect mimics a smaller reflection from the air-snow interface, more subsurface scattering, and a shallower decay. Table 1 shows the errors introduced by the topography into estimates of $\sigma_{\text {surf }}^{o}, \sigma_{\mathrm{vol}}^{\circ}$, and $k_{e}$ when a model of the form

Table 1. Errors in the Retrieval of $\sigma_{\mathrm{surf}}^{\mathrm{o}}, \sigma_{\mathrm{vol}}^{\mathrm{o}}$, and $k_{e}$ Caused by Topographic Undulations With Standard Deviation $10 \mathrm{~m}$ and Autocorrelation Length $5 \mathrm{~km}^{\mathrm{a}}$

\begin{tabular}{lccccc}
\hline $\begin{array}{c}\sigma_{\text {surf }}^{\text {o }} \\
\mathrm{dB}\end{array}$ & $\begin{array}{c}\sigma_{\text {vol, }}^{\mathrm{o}}, \\
\mathrm{dB}\end{array}$ & $\begin{array}{c}k_{e}, \\
\mathrm{~m}^{-1}\end{array}$ & $\begin{array}{c}\sigma_{\text {surf }}^{\mathrm{o}} \text { Error, } \\
\mathrm{dB}\end{array}$ & $\begin{array}{c}\sigma_{\text {vol }}^{\mathrm{o}} \text { Error, } \\
\mathrm{dB}\end{array}$ & $\begin{array}{c}k_{e} \text { Error, } \\
\mathrm{m}^{-1}\end{array}$ \\
\hline 4 & 7 & 0.1 & -1.7 & 0.3 & -0.015 \\
10 & 7 & 0.1 & -0.8 & 1.4 & -0.008 \\
4 & 7 & 0.3 & -1.3 & 0.5 & -0.059 \\
10 & 7 & 0.3 & -0.9 & 1.3 & -0.083 \\
\hline
\end{tabular}

${ }^{a}$ Errors are shown for various combinations of the scattering parameters.

$$
\begin{aligned}
y\left(t ; \sigma_{\mathrm{surf}}^{\mathrm{o}}, \sigma_{\mathrm{vol}}^{\mathrm{o}}, k_{e}, \gamma, \bar{t}\right) \equiv & \frac{\sigma_{\mathrm{surf}}^{\mathrm{o}}}{\gamma \sqrt{\pi}} e^{(t-\bar{t})^{2} / \gamma^{2}}+\frac{\sigma_{\mathrm{vol}}^{\mathrm{o}} c_{\mathrm{ice}} k_{e}}{2} \\
& \cdot \exp \left(\frac{\gamma^{2} c_{\mathrm{ice}}^{2} k_{e}^{2}}{4}-c_{\mathrm{ice}} k_{e}(t-\bar{t})\right) \\
& \cdot\left[1+\operatorname{erf}\left(\frac{(t-\bar{t})}{\gamma}-\frac{\gamma c_{\mathrm{ice}} k_{e}}{2}\right)\right]
\end{aligned}
$$

is fitted to the deconvolution. This model assumes a Gaussian form for the combined convolution of $q, D_{\text {shor }}$, and $D_{\text {long. }}$. As noted above, this will only be appropriate in the flattest regions, when $D_{\text {long }}$ approaches a delta function. Errors for various combinations of scattering parameters are shown.

We found that errors increase as the horizontal scale of the undulations decreases and as their amplitude increases. Smaller errors were obtained for undulations with a decorrelation length of $10 \mathrm{~km}$ and a standard deviation of $10 \mathrm{~m}$ : for decorrelation length $2 \mathrm{~km}$ and standard deviation $3 \mathrm{~m}$ the errors were similar, while for decorrelation length $1 \mathrm{~km}$ and standard deviation $3 \mathrm{~m}$, they were larger. At still smaller scales we found that sastrugi and dunes of a hundred meters or so are sufficiently well averaged to be regarded as part of the shortwavelength topography ( $f_{\text {short }}$ of $(5)$ ), so these will cause little error in the retrieval of the scattering parameters, though they may affect the surface backscatter and the width of the distribution $D_{\text {short }}$ [Legrésy and Rémy, 1997].

\section{Experimental Method and Results}

The surface of ice sheets typically exhibit little expression of bedrock features with scales smaller than the ice thickness, so errors larger than shown in Table 1 are unlikely, except in regions of thinner ice near the margins of the ice sheet, or where wind-induced surface undulations occur on scales of several kilometers [e.g., Fahnestock et al., 2000]. Overall, Table 1 shows that in the presence of undulations typical of an icesheet plateau, it is possible to obtain values of $\sigma_{\text {surf }}^{o}$ and $\sigma_{\text {vol }}^{o}$ to an accuracy of about $1 \mathrm{~dB}$, and the extinction coefficient $k_{e}$ to an accuracy of about $20 \%$, by first obtaining $\bar{r}$ and then fitting the function $y$ defined by equation (19). We used the numerical procedure described in Appendix A to derive $\sigma_{\text {surf }}^{\mathrm{o}}, \sigma_{\mathrm{vol}}^{\mathrm{o}}$, and $k_{e}$ from ERS-1 altimeter echoes recorded over 1195 regions covering the Antarctic plateau.

Plates $1-3$ show the recovered values of $\sigma_{\text {surf }}^{\mathrm{o}}, \sigma_{\mathrm{vol}}^{\mathrm{o}}$, and $k_{e}$, respectively. Values for the ratio of surface to volume scattering and the extinction coefficient are similar in magnitude to those reported by Legrésy and Rémy [1998], who used a different inversion method applied to individual echo waveforms. Generally, the majority of the backscattered power returns from beneath the air-snow interface, with $\sigma_{\mathrm{vol}}^{\mathrm{o}}$ usually exceeding $\sigma_{\text {surf }}^{\circ}$ by $3-10 \mathrm{~dB}$. In Dronning Maud Land $\left(0^{\circ} \mathrm{E}, 76^{\circ} \mathrm{S}\right)$ the two components of the backscatter are both high $(>11 \mathrm{~dB})$ and are more equal. Penetration lengths (inversely related to the extinction $k_{e}$ ) are around 5-10 $\mathrm{m}$ in the snow.

Comparison of our results with field observations and brightness temperatures from microwave radiometers [Surdyk and Fily, 1993] support the interpretations of Rémy et al. [1995], who conclude that extinction $k_{e}$ is dominated by scattering out of the beam by snow grains; $\sigma_{\text {surf }}^{\circ}$ is controlled by reflection from the air-snow interface; and $\sigma_{\mathrm{vol}}^{\mathrm{o}}$ by reflections from di- 
electric discontinuities at the boundaries of density strata within the snowpack. The regions of Dronning Maud Land where $\sigma_{\mathrm{surf}}^{\mathrm{o}}, \sigma_{\mathrm{vol}}^{\mathrm{o}}$, and $k_{e}$ are highest was described by Rundle [1971] as having crust development "so extensive that interpretation (of annual layering) in the field was extremely difficult," with "frequently no fine material at the surface." Thermally emitted microwave radiation is strongly polarized there, an indication of the importance of reflection by density stratification and at the air-snow interface [Surdyk and Fily, 1993]. Nearby regions exhibit lower backscatter: these were described by Rundle [1971] as having surface layers that were "generally soft and fine grained," and they also show weaker polarization effects. We calculated polarization ratios [Surdyk and Fily, 1993] using the $19 \mathrm{GHz}$ brightness temperatures of all 1195 sites as observed by the Special Sensor Microwave/Imager radiometer. These ratios were strongly correlated with $\sigma_{\text {surf }}^{\text {o }}$ (correlation coefficient 0.8 ) and $\sigma_{\mathrm{vol}}^{\mathrm{o}}(0.9)$. Extinction $k_{e}$ was correlated less strongly $(0.4)$ with polarization ratio but follows closely the variations of the vertically polarized gradient ratio (at $18-6.6 \mathrm{GHz}$ ) on the plateau reported by Surdyk and Fily [1993], who relate this quantity to grain size.

Despite the broad agreement in magnitude, there are some notable discrepancies in the spatial distribution between our results and those of Legrésy and Rémy [1998]. Dronning Maud Land and the sector from $120^{\circ}-150^{\circ} \mathrm{E}$ and south of $75^{\circ} \mathrm{S}$ show up as having high extinction $\left(k_{e} \approx 0.3 \mathrm{~m}^{-1}\right)$, while Legrésy and Rémy [1998] report low extinction in these regions (penetration depths of $12 \mathrm{~m}$ or more). The high grain sizes observed in snowpits (data by Surdyk and Fily [1993]), the low thermal microwave emission [Fahnestock et al., 2000], low gradient ratio at $18-6.6 \mathrm{GHz}$ [Surdyk and Fily, 1993], and the strong off-nadir backscatter at Ku-band [Long and Drinkwater, 1999] suggest strong scattering by grains in these regions, consistent with high extinction. However, analysis of echo shape in these regions is also complicated by the presence of long-wavelength (few kilometers) snow dunes with amplitudes of several meters [Fahnestock et al., 2000], and it is not yet certain how these features will affect either of the two inversion methods.

For now, we note that in the estimation of elevation change using satellite crossover data, the spatial variations are of less consequence than temporal changes in echo shape, or effects which depend upon satellite direction, so we analyze these variations in greater detail.

We have performed separate averaging and parameter retrieval for ascending tracks and descending tracks from two time periods (August 14 to September 9, 1992, and July 30 to September 3, 1993). Each of these periods spans a 35-day repeat of the ERS-1 mission, so a full complement of tracks is available for averaging. Insights into changes in the scattering over time and its sensitivity to the satellite's direction of travel can be obtained by comparing the earlier and later data, as well as the ascending and descending passes.

Figure 4 shows the deconvolutions $r$ obtained from a location near Plateau Station $\left(78^{\circ} \mathrm{S}, 37^{\circ} \mathrm{W}\right)$ in 1992 and in 1993 , together with their difference (1992 subtracted from 1993). There has clearly been an increase in backscatter at this site. The backscatter change is localized to the contribution from the air-snow interface (delay times near zero), while scattering from beneath the air-snow interface (delay times greater than zero) is hardly affected. Figure 5 shows that such behavior is typical throughout Antarctica, $\sigma_{\text {surf }}^{\circ}$ decorrelates over the course of the year, while the scattering from the buried snow remains stable. The isolation of the variance in the parameter

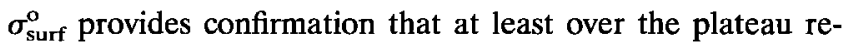
gions studied here, the retrieved parameters are not unduly corrupted by the effects of topography. If they were, a change in the conditions at the air-snow interface would (erroneously) cause changes in the estimates of $\sigma_{\mathrm{vol}}^{\mathrm{o}}$ and $k_{e}$, not just $\sigma_{\mathrm{surf}}^{\mathrm{o}}$. In fact, the observed decorrelation in these parameters is consistent with the effects of speckle noise and instrument gain uncertainties (95\% error bars shown in Figure 5), taken together with the expected sensitivity of the retrieved values of $\sigma_{\mathrm{vol}}^{\mathrm{o}}$ and $k_{e}$ to a change in $\sigma_{\text {surf }}^{\mathrm{o}}$ (which may be estimated from Table 1 for typical undulations).

We did find a few sites in the more undulating terrain of West Antarctica where there is evidence of greater than average control of echo shape by topographic effects. Figure 6 shows deconvolutions from one of these sites $\left(80^{\circ} \mathrm{S}, 95^{\circ} \mathrm{W}\right)$. Here too there has been a change in backscatter between 1992 and 1993, although now the change is seen to extend to larger delay times than Figure 4. There are two possible causes. Either there has been a change in the scattering from beneath the surface at this site, extending to depths of several meters, or there has been a change in the surface backscatter $\sigma_{\text {surf }}^{o}$ and the effect of undulations has redistributed this change to later time delays through convolution with the function $D_{\text {long. The }}$ second explanation seems more likely in view of the undulating terrain in this region, field observations constraining substantial changes in snow properties to the upper centimeters [Alley, 1988], and the absence of any change in the buried snow in flatter regions (Figure 5).

Figure 7 shows a similar correlation analysis for ascending and descending tracks. This time there is little decorrelation in $\sigma_{\text {surf }}^{\mathrm{o}}$ and $\sigma_{\mathrm{vol}}^{\mathrm{o}} k_{e}$ but much greater decorrelation in $k_{e}$. Once again, the successful separation of the variance among just one of the three parameters means that topography has not unduly corrupted their retrieval in these regions. Evidently, the microwave pulse transmitted by the satellite penetrates into the snow to different degrees on ascending and descending passes. A variation in backscatter between ascending and descending passes has been noted by Legrésy and Rémy [1999] and attributed to wind-induced features in the snowpack. Their analysis of waveform shape did not allow them to separate changes in backscatter arising from variations in the radar cross section per unit volume $\sigma_{v}$ from those arising from variations in the extinction coefficient $k_{e}$, and they attributed the effect to variations in $\sigma_{v}$. However, Figure 7 shows that the sensitivity of $k_{e}$ to satellite direction has the controlling influence.

The dependence of $k_{e}$ upon the direction of satellite travel for a nadir-pointing instrument is somewhat surprising. We considered several other possible explanations for our observations. We have tried to reproduce the power and echo shape changes by simulating the echoes that would occur if there were a systematic mispointing of the ERS-1 altimeter but were unable to reproduce either the shape or the amplitude of the discrepancy for plausible mispointing errors and surface slopes.

We also considered diurnal changes in the snowpack as a possible cause. Because the ERS-1 orbit is Sun synchronous, ascending and descending overpasses would each sample any diurnal cycle at a different phase. However, the discrepancy in $k_{e}$ extends to several meters beneath the surface, much deeper than solar radiation, or the diurnal temperature wave, so diurnal changes in firn structure can be ruled out. The changes are also stable from month to month, which is consistent with their being buried out of reach of the wind and Sun and 

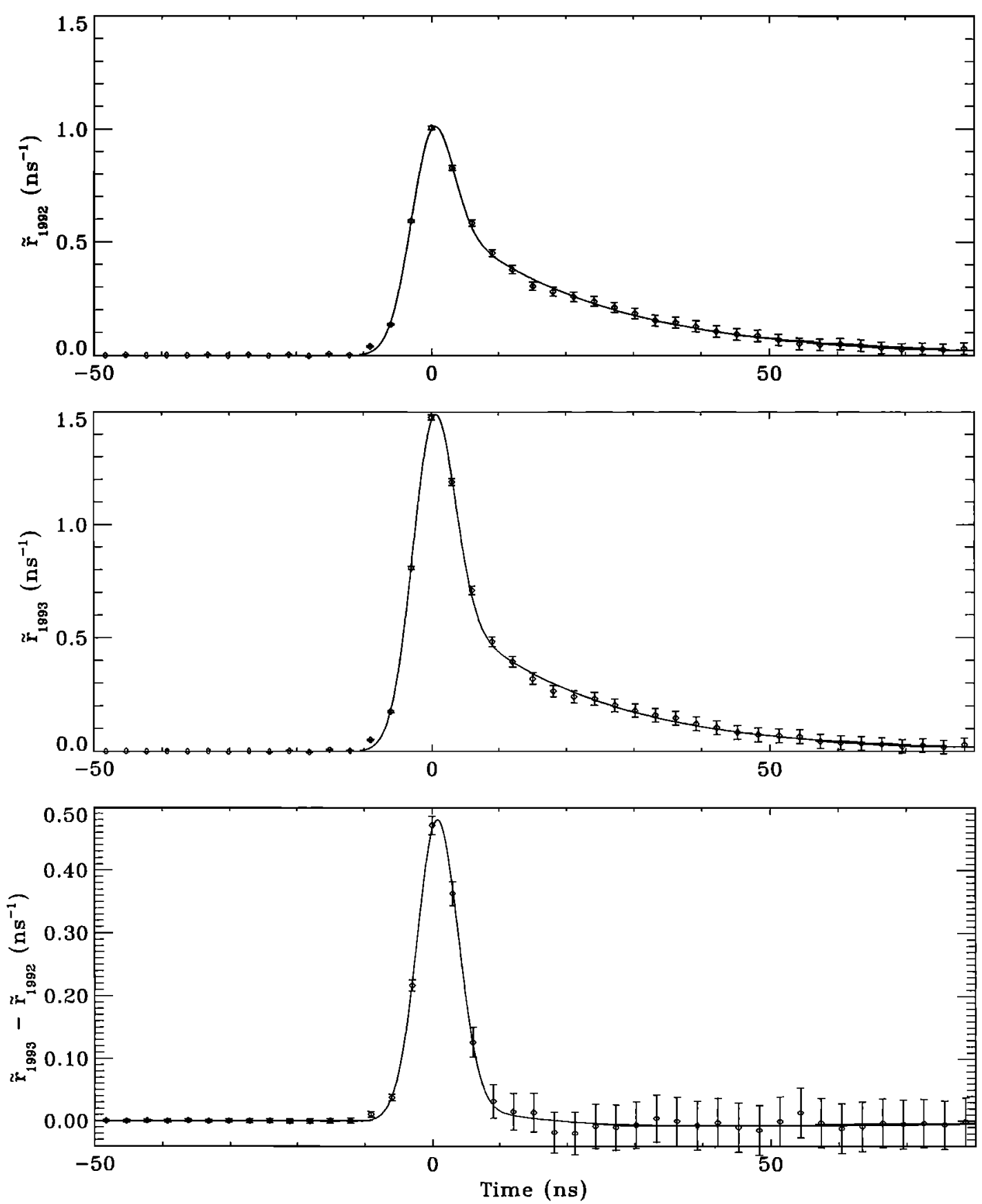

Figure 4. Deconvolutions from near Plateau Station, in September 1992 (top), September 1993 (middle), and their difference (bottom). The change in backscatter is clearly localized at the surface (delay times near zero). Note the Gaussian shape of the difference, indicating that topographic distortion of the echoes is minimal in this region.

inconsistent with their being caused by a diurnal change in snow properties, which would vary seasonally at these latitudes.

The discrepancy between ascending and descending values of $k_{e}$ is largest in Princess Elizabeth Land (especially near $70^{\circ} \mathrm{S}, 90^{\circ} \mathrm{E}$ ) and in Wilkes Land. These regions coincide with those where Rott and Rack [1995] have previously reported a strong anisotropy in the azimuthal dependence of radar backscatter measured away from nadir by the ERS-1 scatterometer. There is undoubtedly a strong azimuthal anisotropy to the snow structure in these regions imparted by the persistent katabatic winds [Goodwin, 1988], and it seems that the extinction of the altimeter pulse is also sensitive to the orientation of this structure. The directional dependence of backscatter observed by Legrésy and Rémy [1999] is a direct consequence of the directional dependence of extinction coefficient, and they have also concluded that the effect is caused by wind-induced features within the snowpack.

Since the ERS-1 altimeter views the snow surface at normal incidence, there is no azimuthal asymmetry brought about by the look direction. However, as noted by Legrésy and Rémy 

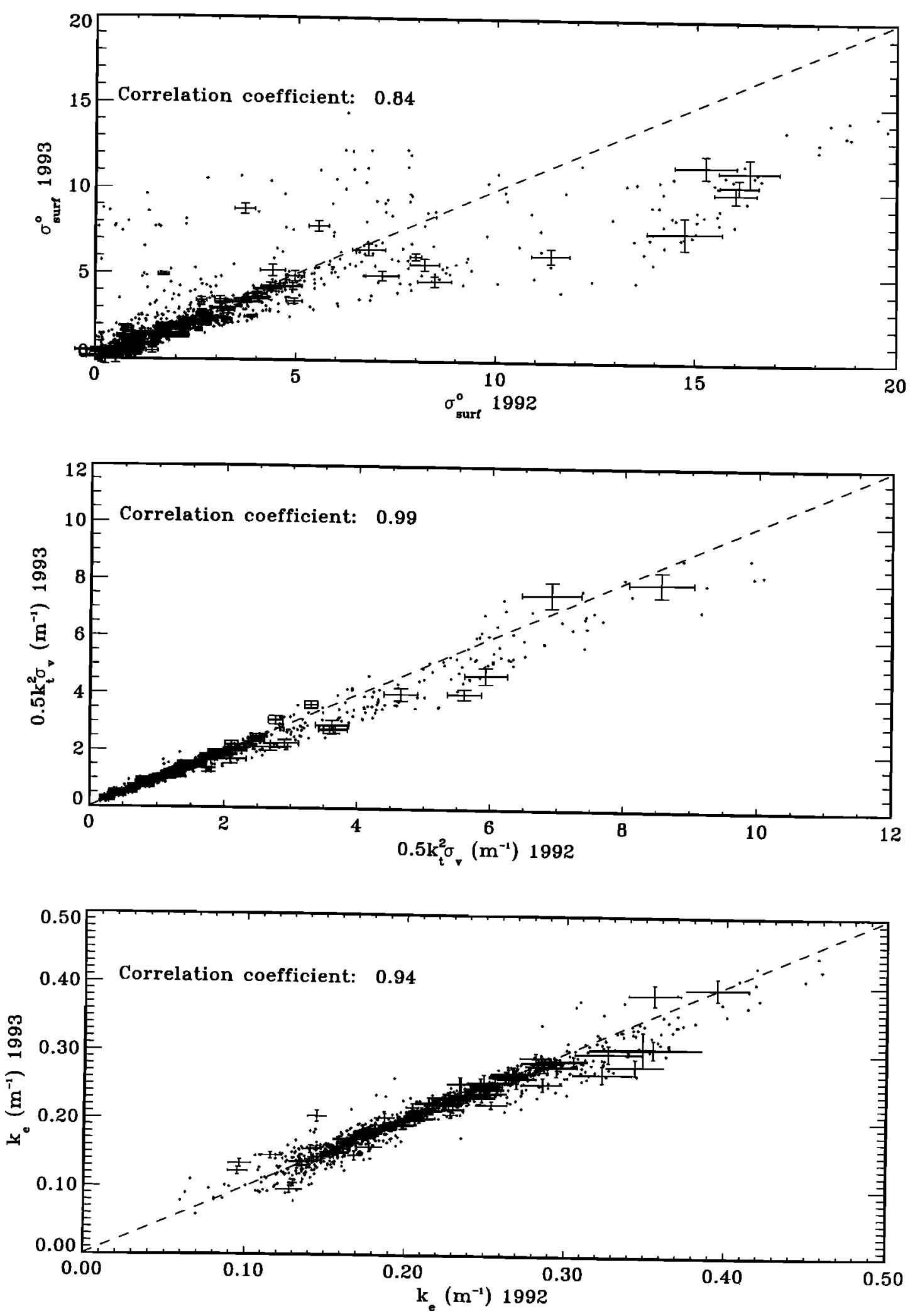

Figure 5. (top) Decorrelation of $\sigma_{\text {surf }}^{\mathrm{o}}$ (middle) $0.5 \sigma_{v} k_{t}^{2}\left(=\sigma_{\mathrm{vol}}^{\mathrm{o}} k_{e}\right)$, and (bottom) $k_{e}$ between September 1992 and September 1993. The decorrelation of $\sigma_{\text {surf }}^{\circ}$ is substantially greater than for the other two quantities. The $95 \%$ confidence intervals for errors caused by speckle noise and instrumental fluctuations are shown on a representative selection of points. 


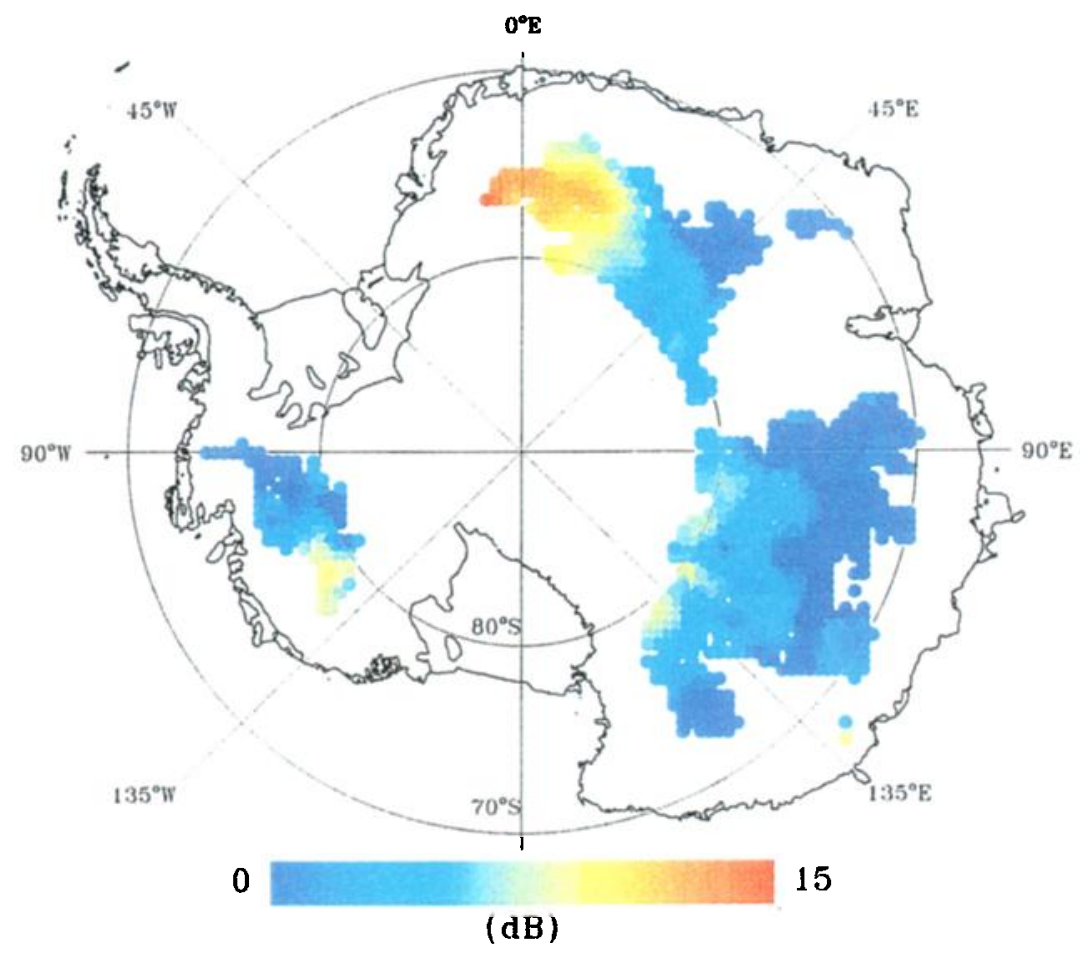

Plate 1. Spatial variations in the surface reflection $\sigma_{\text {surf }}^{\text {o }}$, September 1992.

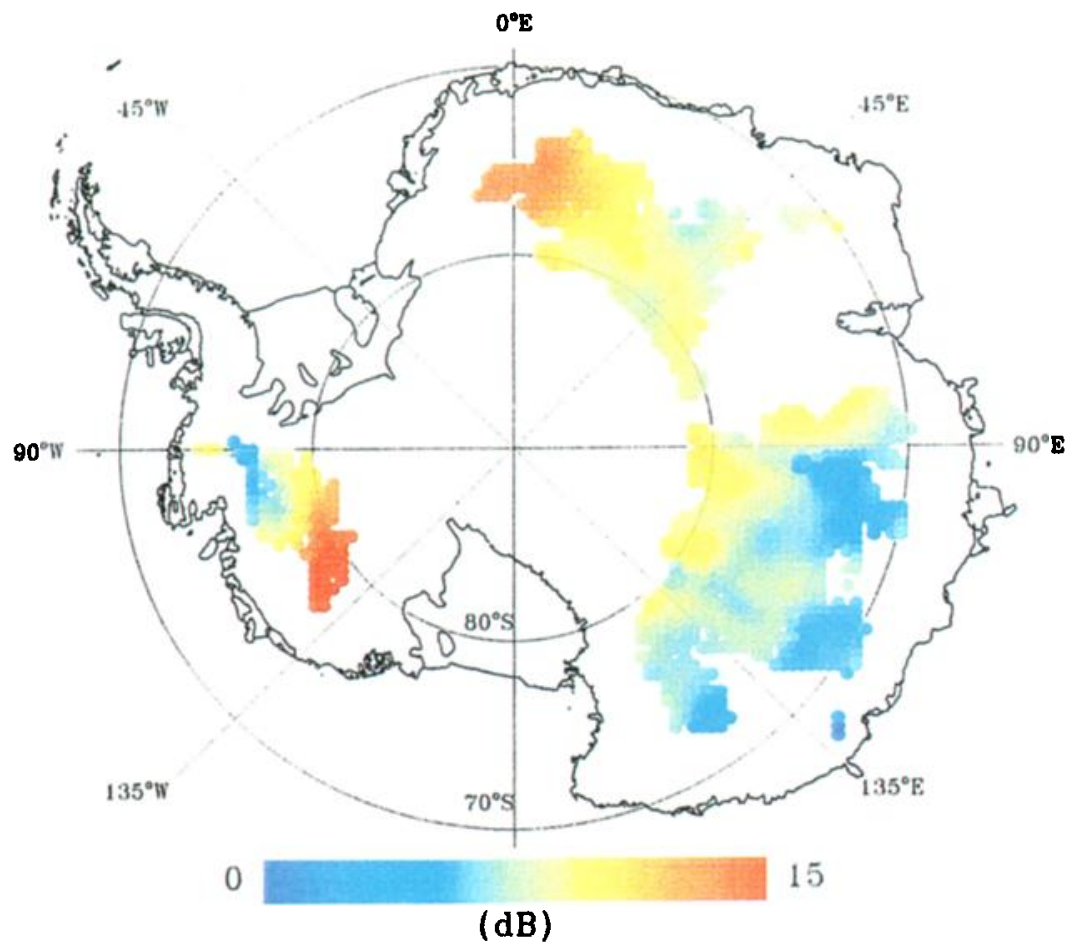

Plate 2. Spatial variations in subsurface scattering $\sigma_{\text {vol }}^{o}$, September 1992. 


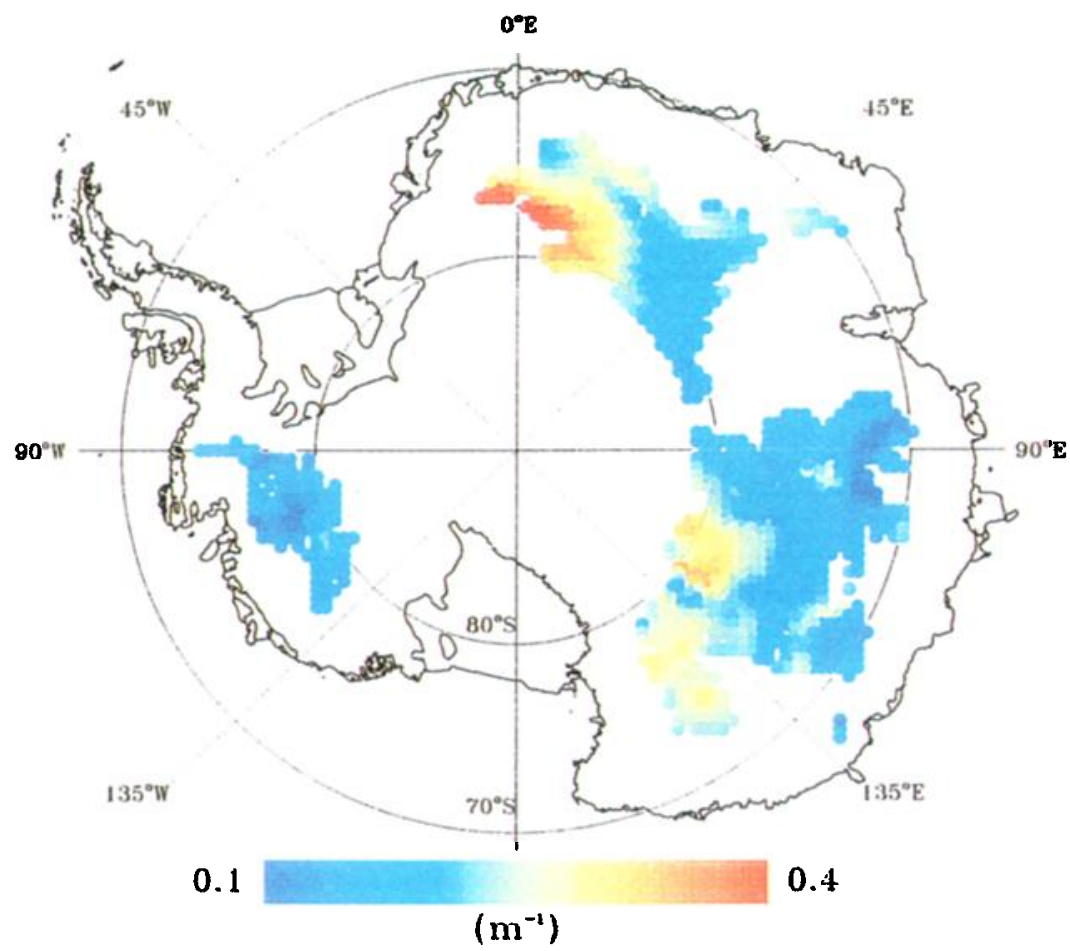

Plate 3. Spatial variations in the extinction coefficient $k_{e}$, September 1992.

[1999], the orientation of the linear polarization vector of the ERS-1 altimeter pulse does change with satellite orientation. The isolation of the effect in the extinction coefficient, rather than the surface or volume backscatter cross section, suggests it is associated with scattering out of the beam, rather than polarization-dependent reflection at layer interfaces. Threedimensional, polarimetric models of microwave propagation in the snow will be needed to investigate the effect further. Such models might also explain the close relationship between this effect and the radar anisotropy measured away from nadir by scatterometery [Legrésy and Rémy, 1999].

Whatever the origin of the asymmetry in $k_{e}$, it is clearly preserved when the snow is buried, at least to depths of a few meters. Even though the processes giving rise to the directional dependence of $k_{e}$ are uncertain, the observation that this dependence is buried and stable over time is important because it allows the error in elevation crossover differences to be minimized, using the approach described in section 5.

\section{Implications for Satellite Measurements of Ice-Sheet Mass Balance}

The observations described in section 4 suggest a way to minimize the errors in measurements of elevation change over ice sheets. We describe this by denoting elevation measurements $H(Z, b)$, where all data within a repeat interval are labeled $Z$, and $b=a, d$ denotes the satellite direction ( $a$ for ascending, $d$ for descending). Any bias due to differences in scattering between ascending and descending tracks can be removed by forming the elevation difference

$$
\begin{aligned}
\Delta H\left(Z_{1}, Z_{2}\right)= & \frac{1}{2}\left[\overline{H\left(Z_{2}, a\right)-H\left(Z_{1}, d\right)}\right. \\
& \left.+\overline{H\left(Z_{2}, d\right)-H\left(Z_{1}, a\right)}\right],
\end{aligned}
$$

where the overbars denote separate spatial averages of each type of crossover difference, and $Z_{1}$ and $Z_{2}$ are orbit repeat periods separated by the interval over which the mass balance measurement is to be determined, typically many years. The separate spatial averages are necessary because most altimeters suffer periods of inoperation and loss of tracking lock, so there is no guarantee that equal numbers of ascending and descending observations are made over an area during each repeat period. This procedure was suggested by Zwally et al. [1989] as a means to reduce the impact of ascending versus descending biases in the satellite orbit solution. It serves equally well to remove ascending versus descending biases in radar penetration, so this procedure is to be encouraged, even when orbit solutions are demonstrably free of ascending versus descending bias.

The use of (20) relies on the temporal stability of the directional dependence in extinction. For long measurements, or in high accumulation areas, where the snow contributing to the echo may be substantially replaced during the measurement interval, this constancy would need to be verified throughout the measurement by confirming that the difference in backscatter measured in ascending and descending directions remains stable.

Another important consideration is that more than one satellite will be required to obtain long time series of ice-sheet elevation changes. Equation (20) relies on the constancy of directionally dependent errors in earlier and later measurements, and this may not be the case if different satellite systems are employed. For instance, the polarization state (circular, linear, etc.), or the orientation of the polarization vector with respect to the direction of travel, may differ between satellites. The orbit of future satellites may also be configured differently so that the satellite does not pass overhead in the same direc- 

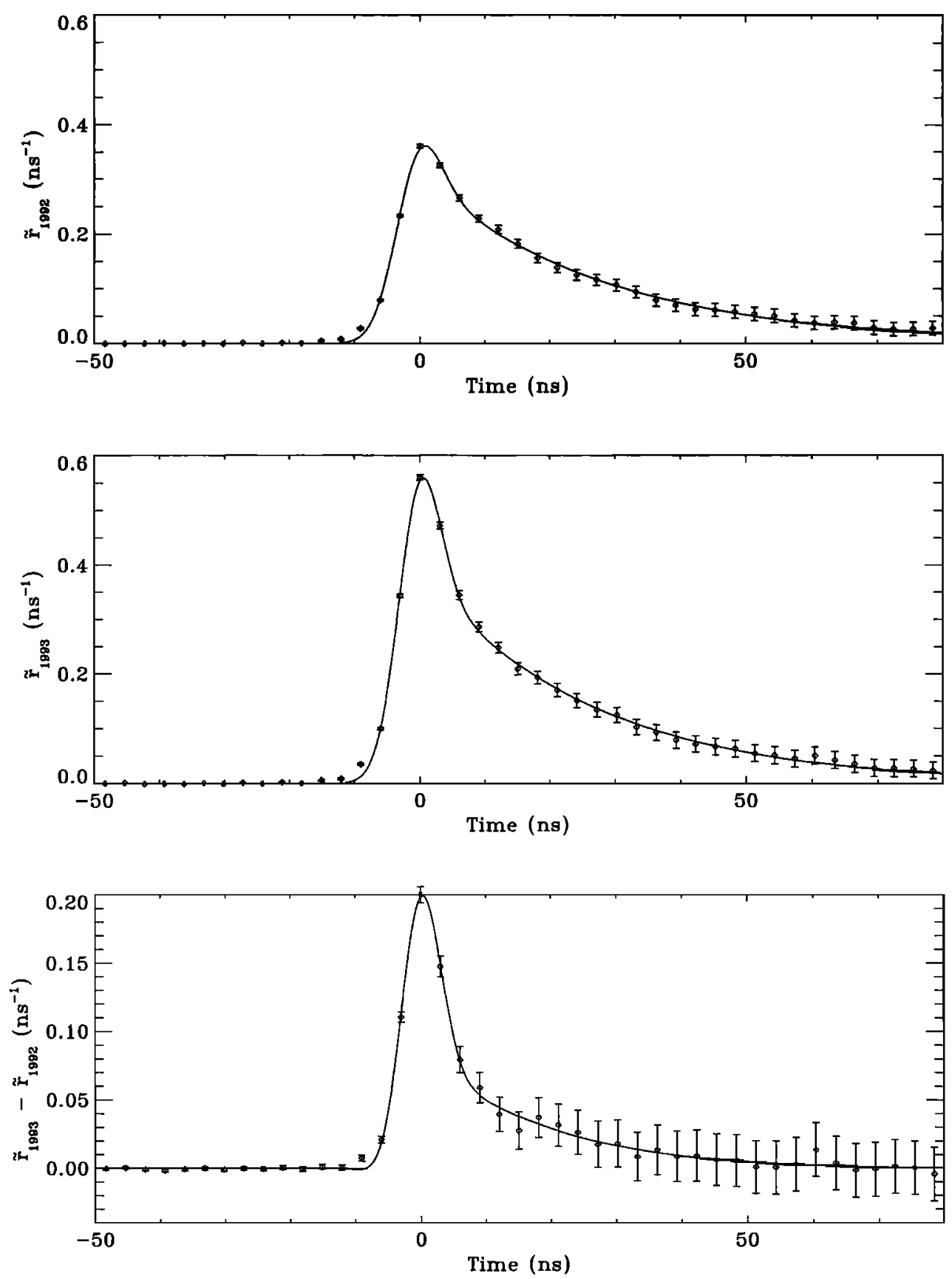

Figure 6. Deconvolutions from West Antarctica in (top) September 1992, (middle) September 1993, (bottom) and their difference. In contrast to Figure 4, the change in backscatter extends to large time delays. Either the scattering from beneath the surface has changed or the echoes in this region are distorted by the undulating topography.

tion as its predecessor. If the extinction coefficient $k_{e}$ is sensitive to the azimuthal orientation of the polarization vector, as the results of section 4 suggest, the intersatellite elevation comparisons might then be in error. There is clearly an incentive to understand the directional dependence of $k_{e}$ further so that different satellite systems can be compared, allowing the longest possible time series of ice-sheet elevation change.

The deconvolution method described in this paper constitutes in large part a practical demonstration of the theory outlined by Wingham [1995] for calculating the elevation change of an ice sheet from observations of the spatially averaged radar echo. A simple change of time origin prior to averaging the echoes would allow calculation of the "datumarrival-averaged" echo considered by Wingham [1995]. Identical deconvolution methods to those described here would then provide the elevation distribution of backscattering from which the average height of scatterers can be estimated [Wingham, 1995].

\section{Summary}

Topographic undulations and microwave penetration both affect the echo shapes recorded over ice sheets. If first-arrival averaging is used to generate an average echo shape over an 

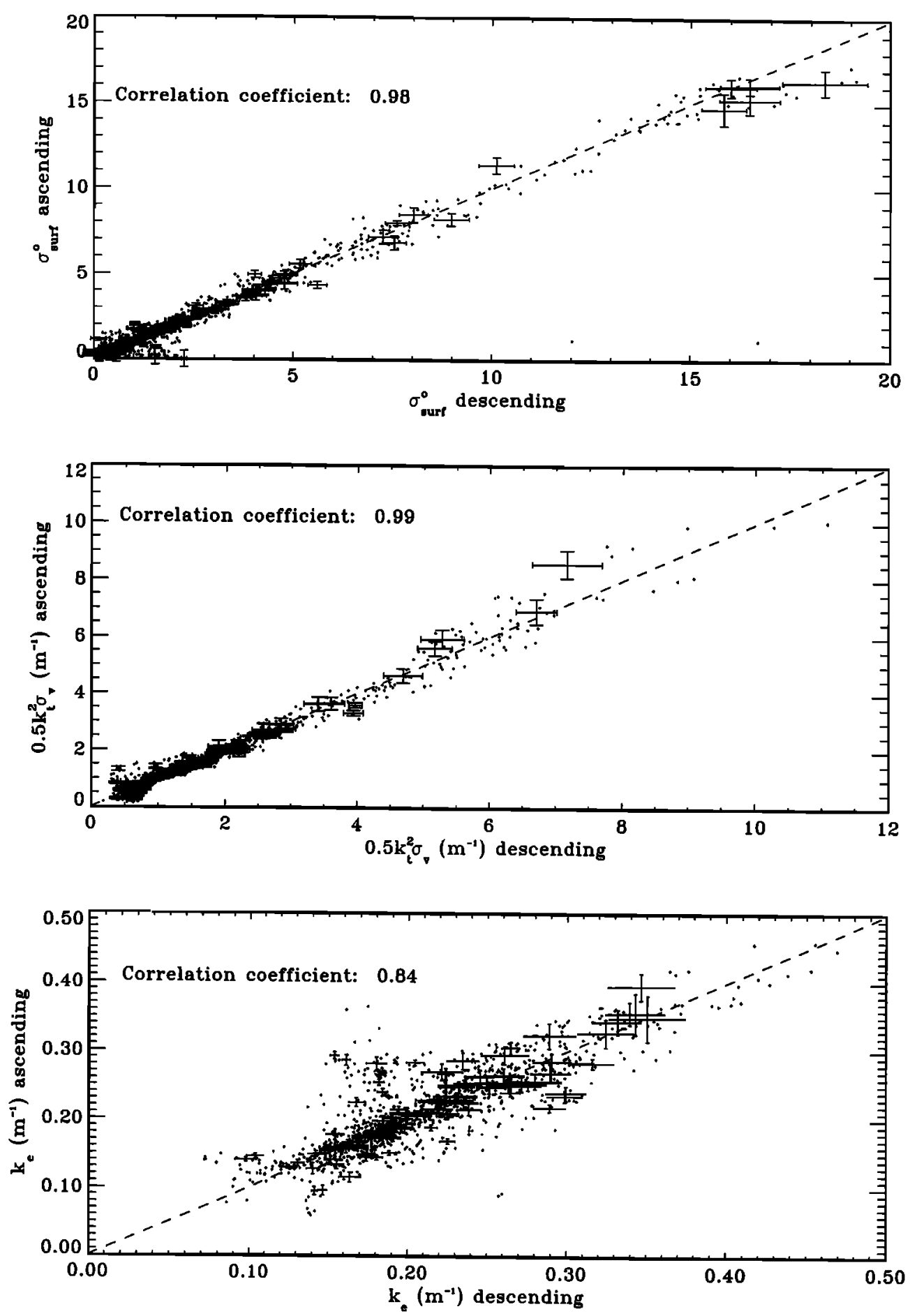

Figure 7. (top) Decorrelation of $\sigma_{\mathrm{surf}}^{\mathrm{o}}$ (middle) $0.5 \sigma_{\tau} k_{t}^{2}\left(=\sigma_{\mathrm{vol}}^{\mathrm{o}} k_{e}\right)$, and (bottom) $k_{e}$ between ascending and descending tracks. The decorrelation of $k_{e}$ is substantially greater than for the other two quantities. The 95\% confidence intervals for errors caused by speckle noise and instrumental fluctuations are shown on a representative selection of points.

undulating region, then topography and penetration can both have very similar effects upon the echo shape, making it difficult to separately determine shape parameters describing the penetration from those describing the topography. In particular, the assumption that topographic undulations distort the echo in the same manner as ocean waves, by convolution with a Gaussian elevation distribution, is likely to lead to errors in all but the flattest regions. These errors will affect estimates of surface elevation, extinction coefficient, surface backscatter and volume backscatter from any algorithm relying upon this assumption. Since the reflection properties of the air snow interface change over time, elevation changes recovered using algorithms which assume a Gaussian convolution model for the effects of topography upon echo shape will also be in error.

Of the echoes we have observed, those from West Antarctica show the most evidence for a mixed effect of penetration and 
topography on echo shape, and we anticipate that this effect will become even more severe as the amplitude and spatial frequency of undulations increase toward the margins. The presence of long-wavelength snow dunes of several meters amplitude may have similar effects. However, over most of the plateau regions of Antarctica, echo shape parameters describing the microwave penetration into the snowpack, and the backscatter from the air-snow interface may be recovered to a useful accuracy from spatially averaged ERS-1 altimeter echoes. These retrievals show that the surface reflection from the air-snow interface is quite variable over time. There is also a strong variation of the extinction coefficient at many sites. The latter variation is temporally stable but depends upon the direction of travel of the satellite. We presume that this is controlled by the orientation of the polarization vector relative to a buried, wind-induced anisotropy in the structure of the snowpack, but detailed modeling or field measurements will be needed to confirm or refute this.

The isolation of the temporal variance in the surface reflection coefficient, and the direction-dependent variance in the extinction coefficient, provides independent confirmation that meaningful echo-shape parameters have been recovered in the plateau regions that we consider here. This isolation is important for the removal of elevation errors brought about by the direction-dependent extinction (section 5). It also allows the correction of errors caused by changes in the surface backscatter [Legrésy and Rémy, 1998; Wingham et al., 1998], to be discussed in greater detail elsewhere.

\section{Appendix A}

It is clear from (15) and (18) that $\bar{r}(t)=\int_{-\infty}^{t} d \tau K_{r}(t-$ $\tau) \bar{p}(\tau)$, so the deconvolution $\bar{r}(t)$ may be viewed as the result of applying an operator defined by the kernel $K_{r}$ to the average echo $\bar{p}$. This appendix describes the numerical implementation of this procedure.

We estimated the origin $t_{0}(M)$ of each echo as the sampling time of the first-echo sample exceeding the $10 \%$ OCOG retracking threshold [Bamber, 1994], then aligned the echoes and averaged them according to (17) to provide $p$, a discrete approximation to the samples $\bar{p}\left(t_{t}\right)$. The ERS-1 radar altimeter averages 50 echoes on board for each record of the echo waveform telemetered to Earth. The number of telemetered waveforms averaged to find $\mathbf{p}$ for each region varied strongly with latitude but was typically several hundred. We used a weighting function $W$ with $100 \mathrm{~km}$ diameter at half maximum. Next, we obtained a discrete function $\mathbf{r}$, which approximates the deconvolution $\bar{r}$ by solving

$$
\mathbf{A r}=\mathbf{p},
$$

where the elements of the matrix $\mathbf{A}$ are given by

$$
a_{\imath j}=\int_{-\infty}^{t_{t}} d \tau I\left(t_{i}-\tau\right) \frac{\sin \left(\omega_{N}\left(\tau-t_{j}\right)\right)}{\omega_{N}\left(\tau-t_{j}\right)},
$$

with $\omega_{N}=\pi / \Delta_{t}$ and $\Delta_{t}=3.02 \mathrm{~ns}$ is the interval between samples. Equations (A1) and (A2) represent a discrete version of (18), derived by using bandwidth-limited interpolation [Press et al., 1986] to approximate the continuous functions $\bar{p}$ and $\bar{r}$ by their discrete counterparts $\mathbf{p}$ and $\mathbf{r}$, and then interchanging the order of summation and integration.

The integral in (A2) was evaluated by Gaussian quadrature, with the function $I$ obtained using (9) and a Gaussian approximation to the antenna gain pattern [Torres, 1990]. In this calculation we used a best fit reference sphere, which was obtained for each region by trial and error minimization of the discrepancies between the sphere and the digital elevation model of Antarctica. If the departure of the surface from the best fit sphere was greater than $100 \mathrm{~m}$ at any point within the weighted region, we made no attempt to deconvolve the echoes, so as not to violate constraints imposed by the use of a linearized model for the echo [Wingham, 1995]. In practice, this constraint limits our retrievals to regions of thicker, gently sloping ice in the interior. The function $I$ depends upon $\Delta$ but not upon $\rho_{S M}$, which cancels upon the integration of (9). We calculated $\Delta$ at the center of each region by assuming the altimeter's boresight is held normal to the WGS84 reference ellipsoid (see Wingham [1995] for the arguments that allow $\Delta$ to be assumed constant over each region). The approximate satellite altitude $h_{0}$ was obtained by calculating the closest sphere-to-satellite distance for a representative selection of data over each region. Atmospheric losses were assumed small, so $L_{s} \approx 1$.

Equation (A1) was solved by singular value decomposition [Press et al., 1986]. The parameters $\sigma_{\text {surf }}^{\mathrm{o}}, \sigma_{\mathrm{vol}}^{\mathrm{o}}, k_{e}, \gamma$, and $\bar{t}$ were estimated by fitting the function defined by (19) to the deconvolution using the Levenerg-Marquadt method [Press et al., 1986]. We used $c_{\text {ice }}=2.35 \times 10^{8} \mathrm{~ms}^{-1}$, which is appropriate for typical snow densities [Matzler, 1996].

\section{Notation}

$a$ ascending tracks,

$\mathbf{A}, a_{\imath}$ matrix and coefficients relating $\mathbf{p}$ and $\mathbf{r}$,

$b$ satellite direction of travel,

$c, c_{\text {ice }}$ speed of light in vacuum and ice, respectively,

$d$ descending tracks,

$D_{\text {Iong }}$ convolution term arising from long-scale topography,

$D_{\text {short }}$ convolution term arising from short-scale topography,

$\Delta$ incidence angle between boresight and sphere normal,

$\Delta_{t}$ sampling interval in time delay,

$E_{t}$ total energy transmitted per pulse,

$\eta$ curvature factor,

$f$ surface elevation above reference sphere,

$f_{\text {long }}$ long-wavelength component of $f$,

$f_{\text {short }}$ short-wavelength component of $f$,

$g$ antenna gain pattern,

$\gamma$ leading edge width parameter,

$h_{0}+\delta h(M)$ satellite altitude above reference sphere,

$H$ elevation measurement,

$\Delta H$ measured elevation change,

$I(t)$ impulse response of reference sphere,

$I_{M}(M, t)$ impulse response of surface $f_{\text {long }}$ at $M$,

$k_{e}$ extinction coefficient,

$k_{t}$ transmission coefficient,

$L_{s}$ atmospheric loss,

$\lambda$ wavelength in free space,

$M$ closest point on reference sphere to altimeter,

$N$ closest point on reference sphere to scattering point, 
$O$ center of reference sphere,

$\omega_{N}$ Nyquist frequency of time delay sampling,

$\bar{p}(t), \mathbf{p}$ first-arrival-averaged echo and its discrete approximation,

$p(M, t)$ individual echo recorded at $M$,

$q(t)$ normalized effective transmitted power envelope,

$\bar{r}(t), \mathbf{r}$ deconvolution and its discrete approximation,

$R, \rho, \phi$ spherical coordinates of $N$, $\rho_{S M}$ boresight azimuth,

$s$ time delay distribution for penetration,

$\sigma^{\circ}$ total backscatter cross section,

$\sigma_{\text {surf }}^{\circ}$ surface backscatter cross section,

$\sigma_{v}$ backscatter cross section per unit volume,

$\sigma_{\text {vol }}^{\circ}$ depth-integrated volume backscatter,

$t$ time delay since transmission,

$\bar{t}$ leading edge time delay obtained from model fit,

$t_{0}(M)$ time delay of first return,

$\theta$ angle from boresight,

$W$ spatial weighting function for echoes,

$y$ model fit to deconvolution.

Acknowledgments. This work was in part supported by the U.K. Natural Environmental Research Council under grant GR3/8748. We would like to thank Judith Proud and Justin Mansley for assistance in processing ERS-1 data. SSM/I data were supplied by the National Snow and Ice Data Center, Boulder, Colorado, and ERS-1 data by the European Space Agency.

\section{References}

Alley, R. B., Concerning the deposition and diagenesis of strata in polar firn, J. Glaciol., 34(118), 283-290, 1988.

Bamber, J. L., Ice sheet altimeter processing scheme, Int. J. Remote Sens., 15(4), 925-938, 1994.

Brenner, A. C., R. A. Bindschadler, R. H. Thomas, and H. J. Zwally, Slope-induced errors in radar altimetry over continental ice sheets, J. Geophys. Res., 88, 1617-1623, 1983.

Brown, G. S., The average impulse response of a rough surface and its applications, IEEE Trans. Antennas Propag., AP-25(1), 67-74, 1977

Davis, C. H., and R. K. Moore, A combined surface- and volumescattering model for ice-sheet radar altimetry, J. Glaciol., 39(133), 675-685, 1993.

Davis, C. H., C. A. Kluever, and B. J. Haines, Elevation change of the Southern Greenland Ice Sheet, Science, 279, 2086-2088, 1998.

Fahnestock, M. A., T. A. Scambos, C. A. Shuman, R. J. Arthern, D. P. Winebrenner, and R. Kwok, Snow megadune fields on the East Antarctic Plateau: Extreme atmosphere-ice interaction, Geophys. Res. Lett., 27(22), 3719-3722, 2000.

Féménias, P., F. Rémy, R. Raizonville, and J. F. Minster, Analysis of satellite-altimeter height measurements above continental ice sheets, J. Glaciol., 39(133), 591-600, 1993.

Goodwin, I. D., Firn core data from shallow drilling investigations in eastern Wilkes Land, East Antarctica, Tech. Res. Notes 65, Aust Natl. Antarct. Res. Expeditions, Antarct. Div., Kingston, Tasmania, Australia, 1988

Joughin, I., D. P. Winebrenner, M. Fahnestock, R. Kwok, and W.
Krabill, Measurement of ice-sheet topography using satellite radar interferometry, J. Glaciol., 42(140), 10-22, 1996.

Legrésy, B., and F. Rémy, Altimetric observations of surface characteristics of the Antarctic ice sheet, J. Glaciol., 43(144), 265-275, 1997.

Legrésy, B., and F. Rémy, Using the temporal variability of the radar altimetric observations to map the surface characteristics of the Antarctic ice sheet, J. Glaciol., 44(147), 197-206, 1998.

Legrésy, B., and F. Rémy, Different ERS altimeter measurements between ascending and descending tracks caused by wind induced features over ice sheets, Geophys. Res. Lett., 26(15), 2231-2234, 1999.

Lipa, B. J., and D. E. Barrick, Ocean surface height-slope probability density function from SEASAT altimeter echo, J. Geophys. Res., 86, 10,921-10,930, 1981.

Long, D. G., and M. R. Drinkwater, Cryospheric applications of NSCAT data, IEEE Trans. Geosci. Remote Sens., 37(3), 1671-1684, 1999.

Martin, T. V., H. J. Zwally, A. C. Brenner, and R. A. Bindschadler, Analysis and retracking of continental ice sheet radar altimeter waveforms, J. Geophys. Res., 88, 1608-1616, 1983.

Matzler, C., Microwave permittivity of dry snow, IEEE Trans. Geosci. Remote Sens., 34(2), 573-581, 1996.

Partington, K. C., J. K. Ridley, C. G. Rapley, and H. J. Zwally, Observations of the surface properties of the ice sheets by satellite radar altimetry, J. Glaciol., 35(120), 267-275, 1989.

Press, W. H., B. P. Flannery, S. A. Teukolsky, and W. T. Vetterling, Numerical Recipes: The Art of Scientific Computing, Cambridge Univ. Press, New York, 1986.

Rémy, F., P. Féménias, M. Ledroit, and J. F. Minster, Empirical microwave backscattering over Antarctica: Application to radar altimetry, J. Electromagn. Waves Appli., 9(3), 463-474, 1995.

Ridley, J. K., and K. C. Partington, A model of satellite radar altimeter return from ice sheets, Int. J. Remote Sens., 9(4), 601-624, 1988.

Rott, H., and W. Rack, Characterisation of Antarctic firn by means of ERS-1 scatterometer measurements, in Proceedings of IGARSS 95, Inst. of Electr. Electron. Eng., New York, 1995.

Rundle, A. S., Snow accumulation and stratigraphy on the Antarctic plateau, Antarct. Res. Ser., 16, 239-255, 1971.

Skolnik, M. I., Radar Handbook, McGraw-Hill, New York, 1970.

Surdyk, S., and M. Fily, Comparison of the passive microwave spectral signature of the Antarctic ice sheet with ground traverse data, Ann. Glaciol., 17, 161-166, 1993.

Torres, R., ERS-1 Radar Altimeter Antenna E.M. Test Report, Eur. Space Agency, Paris, 1990.

Wingham, D. J., A method for determining the average height of a large topographic ice sheet from observations of the echo received by a radar altimeter, J. Glaciol., 41(137), 125-141, 1995.

Wingham, D. J., A. L. Ridout, R. Scharoo, R. J. Arthern, and C. K. Shum, Antarctic elevation change, 1992 to 1996, Science, 282, 456458, 1998.

Zwally, H. J., A. C. Brenner, J. A. Major, R. A. Bindschadler, and J. G. Marsh, Growth of Greenland ice sheet: Measurement, Science, 246, 1587-1589, 1989.

R. J. Arthern, British Antarctic Survey, Natural Environment Research Council, High Cross, Madingley Road, Cambridge, CB3 0ET, England, UK. (rart@bas.ac.uk)

A. L. Ridout and D. J. Wingham, Department of Space and Climate Physics, University College London, Gower Street, London, UK, WC1E 6BT.

(Received February 4, 2001; revised July 11, 2001; accepted August 15, 2001.) 